\title{
دراسة تحليلية عن كتاب "امثلتي ه" للحاج توفق الحكيم
}

\author{
Jamaluddin Shiddiq ${ }^{1}$, Ummul Ma'rifah \\ ${ }^{1}$ Institut Agama Islam Negeri Ponorogo
}

Jamaluddin@iainponorogo.ac.id

\begin{abstract}
The purpose of this study was to find out: the presentation of the book Amtsilati part 5 by Haj Tawfiq al-Hakim and the presentation the method evaluation of it. This research is a type of library research. The results of this study are: (1) In terms of presentation of material, the material chosen is in accordance with the learning objectives. In terms of the level of ability of students, this book is suitable to be taught to elementary level students. As for the ease of learning, there are significant problems, namely the example from the Qur'an will be difficult for non-Arabic students. Besides the material, this book uses two examples of gradations, namely collective gradations and sequential gradations. In presenting materials, this book uses three procedures: priority procedures, drawing procedures, and context procedures. As for the addition of the presentation, it was considered not good. For material review, this book contains several exercises for material repetition. (2) In terms of evaluation, amtsilati 5 uses a type of formative structural evaluation. Various forms of evaluation are used, such as form training, word composing exercises, word completing exercises, application exercises, repetition training and meaning giving exercises.
\end{abstract}

Keywords: amtsilati, content analysis, book review

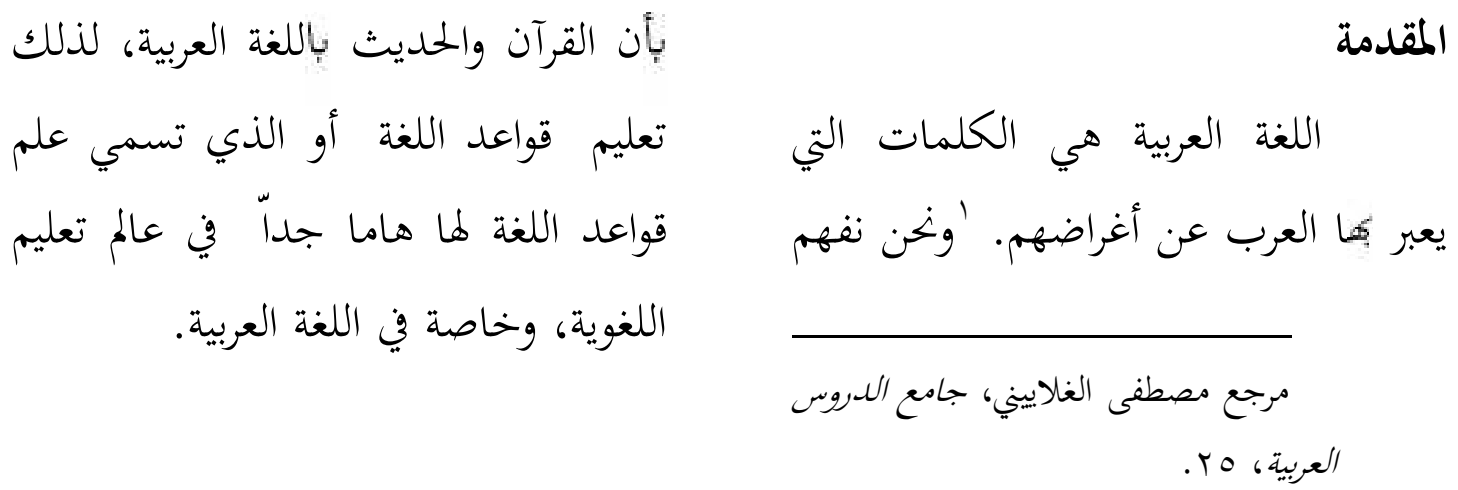


كتاب النصّ بتعل التلاميذ على فهم اللغة العبية بالسهولة.

من عديد كتاب النصّ اللغة العربية، "أمثلتي" هو الكتاب الذي يتألف الحاج توفيق الحكيم جيبارا، الذي لئي يبحث في علم القواعد العربية، لبعض

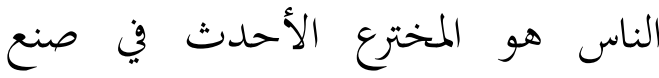
التعليم قواعد اللغة العربية بعملية. هذا هو فتان الباحثة للأداء البحث دراسة تحليلية لكتاب" امثلتي". ستبحث الباحثة ويحليل محتوى في كتاب الأمثلتي ه تأليف الحاج توفيق الحكيم بالتفصيل.

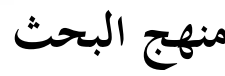

المدخل الذي استعملته الباحثة

هو المدخل الكيفي ـ المدخل الكيفي هو المدخل فيه تطلب الباحثة البيانات بطريقة

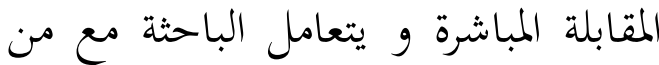

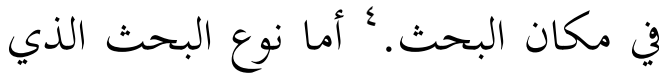

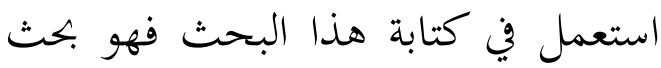

Metode شمس الدين و أخرون، Penelitian Pendidikan Bahasa

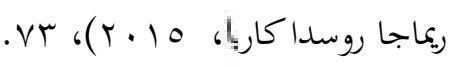

كانت اللغة العربية هي احدى المواد الدراسية الهامة في العالم التربوي

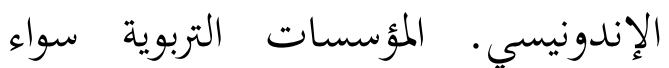
كانت حكومية أم أهلية تدرس اللغة العربية كما تدرس المواد الأخر. و و خاصة في المؤسسات التربوية الإسلامية كانت اللغة العربية مادة دراسية ضرورية لجميع

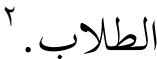

يشكل الكتاب التعليمي عنصرا أساسيا من مكونات المنهج، فهو إحدى ركائزه الأساسية في أي مرحلة تعليمية، قمن محتوه اللغوي و الثقافي تتحقق الأهداف التي تريد تحقيقها من العملية التعليمية، بالإضافة إلى المكونات الأخرى للمنهج من أنشطة و طرق تدريس. المشكلة الآن على الرغم الكتاب المدرسي

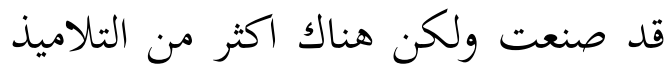
الذي يتعلّم اللغة العربية بالسعوبة. ينبغي r بنس المراجع، 101.

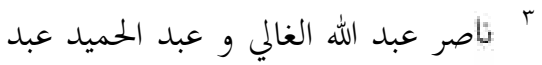

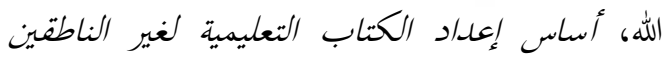
بالعربية ( القاهرة: دار الإعتصام، بغير سنة)، V. V. 
مصدر الوثيقة و الكتب و الجرائد و المجلات و غيرها.

كيفية تحليل البيانات التى استعملتها الباحثة هو تحليل المحتوى. تحليل البجتوى هو التحليل الذي لنيل الخبر او المعلومات من المحتوى الذي يظهر بشكل الرمز.^ يشمل التحليل المحتوى على السعي و تصنيف الرمز في الإتصال، و يستخدم المقياس في التصنيف، و يستخدم اسلوب التحليل الخاص في جعل بي بل بل النبوءة.

\section{نتائج البحث ومناقشتنها}

قد عرفنا بأنّ حجة في تأليف

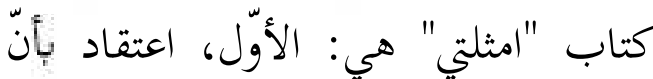
يقرأ كتاب التراث هو صعوبة، و يحتاج إلى

Metode Penelitian هداري نواوي

. . Bidang Sosial

Metode الال الدين رحمة

( Penelitian Komunikasi

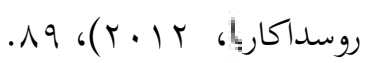

Analisis Data برهان بوعين، Penelitian Kualitatif

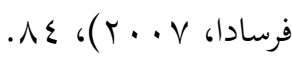

المكتبي. وهو أنشطة البحث الذي تفعل الباحثة و بتمع البيانات من المطبوعات, إمّا في المكتبة أو في في مكان الأخر. المطبوعات المستخدمة ليس في الكتب فقط ولكن من التوثيق و البحلات و الجريدات و الكتابية. $\quad$ بحث تحليل المضمن هو البحث الذي يفعل بالمنظم علي النص و الوثيقة كمصادر البيانات. اسالب مع البيانات مي الأسالب التي تستخدم الباحثة لنيل البيانات. وأسلوب جمع البيانات في هذا البحث هو الأسلوب الوثيقي هو طريقة جمع البيانات بطابقة و تصنيف المواد الكتابي المتعلقة بمسألة البحث. إمّا في

Metode Penelitian هدري نواوي، Bidang Sosial

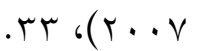

Metodologi بنور الذرية،

Penelitian Sosial Dan Pendidikan (جاكارنا: بومي اكسارا، 9. . ب)، 0. 
الكتاب النص على هذا النهج. و كذالك كتاب " امثلتي ه".

في هذا البحث يحلل الباحثة كتاب "امثلتيه" بتحليلية التي عبر عنها يكا لبحل بجكي وهي: اختيار المواد و تدرّج المواد و تقديم المواد و تكرار المواد.

1. اختيار المواد في كتاب "امثلتي ه" المراد المتاد اختيار المواد هي اختيار

مواد قواعد اللغة التربوي من مصادر القواعد الوصفي. اختيار المادة هي تصن تعتبر هامة في تطوير و تحليل المواد من تعليم اللغة. حتى في غاية الأهمية، بكيث يتم تحديد جودة تصميم التعليم من خلال نوعية عمل الإختيار. و وفي هذا البحث سوف يظاهر الباحثة الصورة التي تظاهر المظاهر في جانب اختيار المواد في هذا الكتاب. أنظر إلى جلى انيار

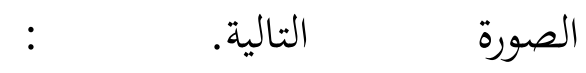

قدرة اتقان قواعد اللغة العربية التي يبحث في كتب الثقيلة مثل الفية ابن مالك و ولاعد

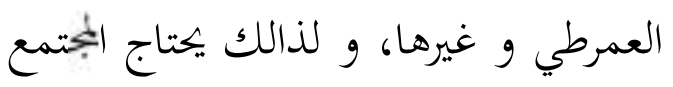

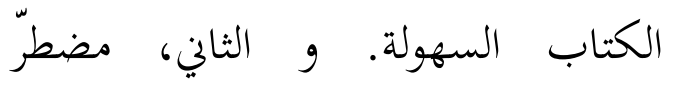
باختراع طريقة قراءة القرأن ب "قراءئ" في التئي سيمارانج، حتى يحتاج الطريقة مثلها،

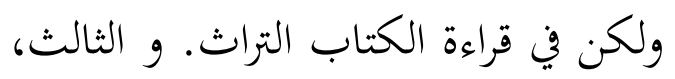

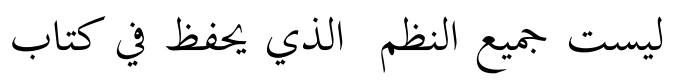
التزاث متعلق بقواعد اللغة العربية يستخدم في قراءة كتاب التراث، حتي يجتاج إيجازه فتط. تقديم كتاب "امثلتي ه" للحاج توفق الحكيم الكتاب هو مصدر من مصادر

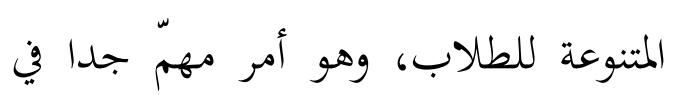

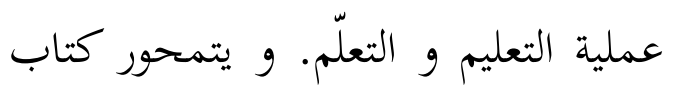
النص لتسهيل الطلاب في تعليم درس اللغة العربية. نص الكتاب مساعدة في التعليم و التعلم. و ينبغي في اعداد 


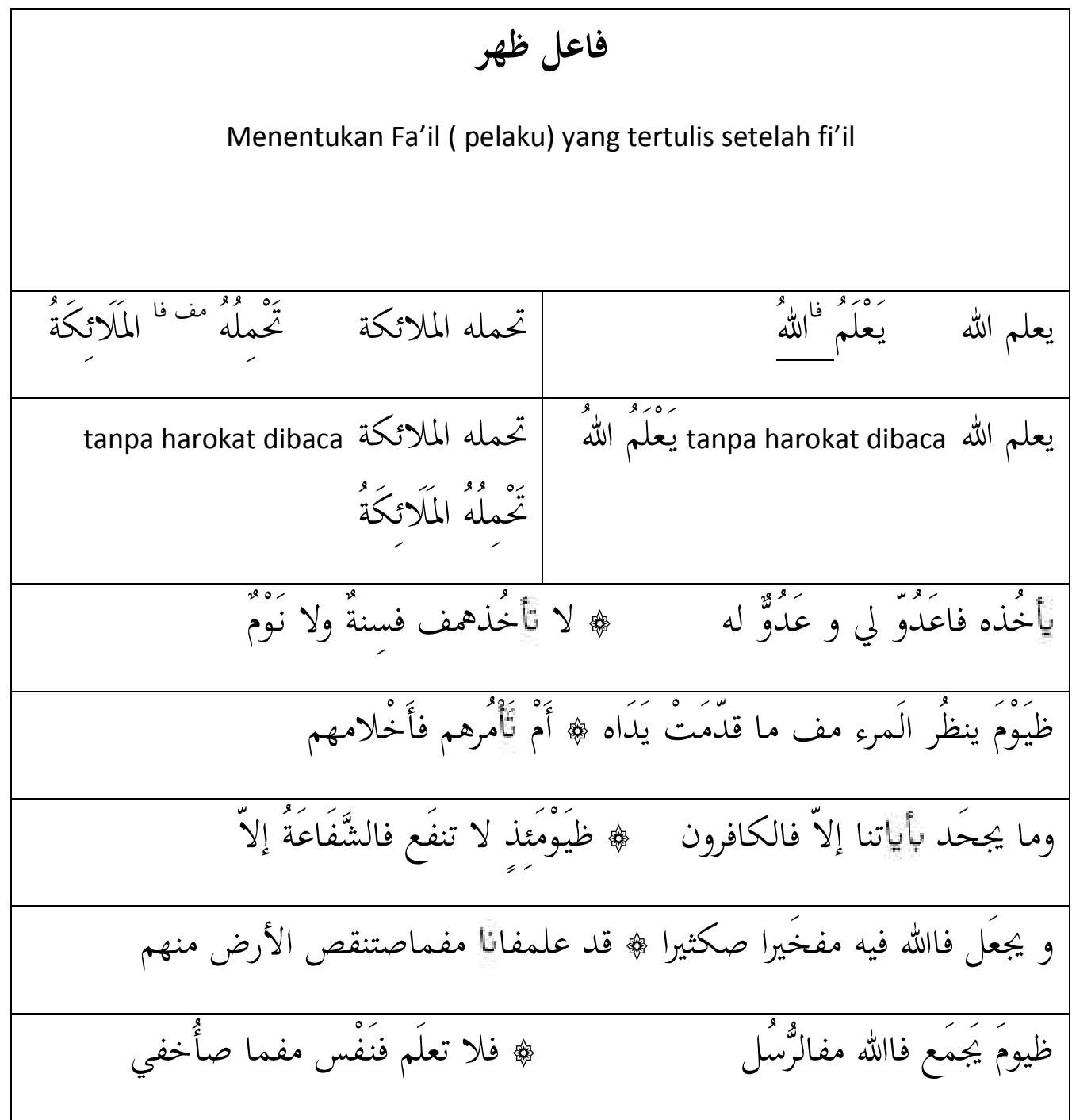

تعليم اللغة إلى هدف

التكامل و هدف الفعال.

تحتاج أهداف تعليم اللغة إلى

معرفته قبل إختيار المواد

للتعليم قواعد اللغة. لأنّ
قال مجكي، كان لإختيار

المواد مبادئا الذي يشكّل

الإختيار و هي:

.

غرض ت تعليم اللغة

مختلف. و يتكون أهداف 
يستعمل المبحث "الفعل" و

هو المواد يوافق أن يتعلم لطلاب الإبتدائ.

$$
\text { . }
$$

في عملية تعليم، كل

تعلم اللغة يصميم بوقت

المحدد، كان التخصيص يملدد

على كمية الوقت الذي سيتمّ

التعليم الذي يتعلمه. لذالك

الأمر، وجب على مؤلف

الكتب لتوزيع الوقت في هذا

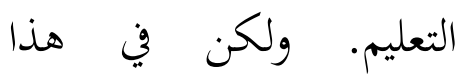

الكتاب الؤلف لا يشرح

الوقت، الوقت في تعليم هذا

$$
\text { الكتاب هو حر. }
$$$$
\text { . سهولة للتعليم }
$$

تتعين المواد لها

سهولة في تعليم اللغة بجوانب

محددة التي وجدت في عملية

تعليم اللغة. منها الأمور

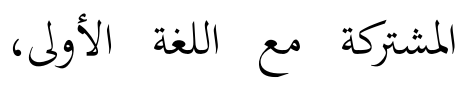

اختلاف الغرض في تعليم

اللغة سيطالب مواد مختلفة.

عند رأيّ الباحثة ذالك

النص من صورة السابقة التي

يشمل الأمثلة المتنوعة من من لئ لئي

الجمل و المفردات المستمدة

من أية القرأن الكريم وهي

وفقا بالغرض من التعلم

الكتاب, وهو لتسهيل

طلاب الإبتدائ لتعليم قراءة

كتاب التراث و فهم القرأن

الكريم بالأعمق.

. مستوى كفاءة من الطلاب

على نطاق واسع

مستوى اللغة على ثلاثة،

وهي: المستوى الأساسي و

المتوسطي و المتقدمي. وفقا

بما يكتب في صفحة الغلاف

لأنّ هذا الكتاب لطلاب

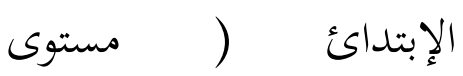

الأساسي). وفي هذا الكتاب 
في هذا الكتاب يستعمل

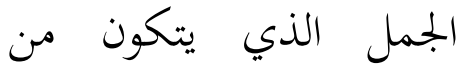

الكلمة الكثيرة من أية القرأن

$$
\text { الذي لها أدبية عالية. }
$$

و أما في جانب مناسبة مع اليناليها

اكتساب اللغة ، في عموم الطلاب

يستطيعون على نموط او الجملة

الأساسية . هذا الحصول على لى الحملى

التحول بناء الجملة بعدها. و هذا

يعني أن في اختيار المواد، و تعليم

أنمط الجملة أولى. و بناء على ذلك آلك الكيار

إختيار المواد في هذا الكتاب يبدأ

بلمواد " فعل المضارع" وبعد ذلك

$$
\text { فهو فعل الأمر. }
$$

و في جانب اللغاوية،

$$
\text { تنقسم إختيار المواد إلى أربعة: }
$$$$
\text { . }
$$

في اختيار المفردات يجب

للمؤلف الكتاب ليهتم" الترديد

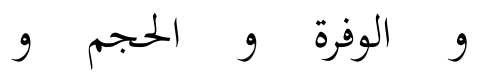

السيكولوجية و التعليمي في
وقواعد اللغة النظام، و

البساطية، و مناسبة مع الكعاطية

اكتساب اللغة.

وفقا عند الباحثة بأنّ هذا

الكتاب يستعمل ثلاث لغات وهي

لغة العربية و لغة الإندونيسية (اللغة

الأولى) و لغة الجاوية (اللغة الأولى)

لأن في قراءة الكتب التراث في

جاوية يراد المعنى بمعنى الجاوية و

$$
\text { الإندونسية. }
$$

وفي قواعد اللغة النظام، في

هذا الكتاب المثال يبيحث الفعل

المضارع، أولا يبحث في تعريف فعل

المضارع و يستمر يبحث في كيفية

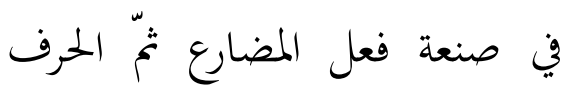

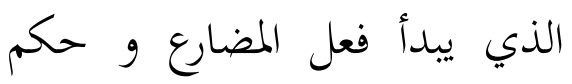

فعل المضارع في اعرابه. وهو وفقا

بالنظرى عن سهولة التعليم.

وامّا

البساطية، وهي تتكون من

الإثنين و الثلاث الكلمة. 
النص. مستوى صعوبة عن

النص لا يحلدد بكمية الكلمة

و التركيب الذي يعرفه، لكنّ

يحدد أيضا بأصول المنطق.

يعطي هذا الكتاب النص من

أية القرأن الذي سيكون

صعوبة خاصة عندما تواجه

مع الطلاب غير العرب، و

خاصة إندونيسيا.

• تدرج المواد في كتاب "امثلتي ه"

انطلاقا محا سبق، كان الغرص

من تأليف كتاب "امثلتي" هو

تساعد لسهل الطلاب المبتدئين

ليقرأ كتاب التراث و فهم القرأن

بالأعمق. ينبغي لسهل الطلاب،

يصميم المواد بإجراء التدرّ.

التدرج هو يسلسل مادة

القاعدة قواعد اللغة الذي سيعلم و

مختار. التدرج هو مرحلة المهارة بعد

مصنف القواعد التربوية قد اثبت

مادته من مصادر القواعد اللغة
اختيار المفردات

الكتاب يشرح و يعي

الأمثلة من الوزن الفعل و هو

من جانب الترديد كثير

استخدمه في عملية التعليم

اللغة العربية. لذالك يوافق

اختيار المفردات ي هذا

الكتاب بالإطار النظري عند

الباحثة.

· اختيار التركب .

ينبغي لإختيار التركيب

أن يهتم حقيقة السيكولوجية

و التعليمي عن الطلاب

(الإبتدائ). ولكن. في هذا

الكتاب بعطي الأية من

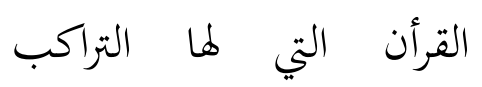

المتنوعة الصعوبة عند الطلاب

المبتدئين.

$$
\text { . }
$$

ينبغي لإختيار النص أن

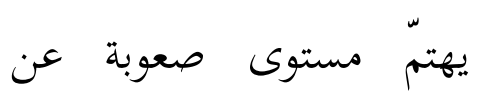


يسند بمبداء اساسية السيكولوجية

التعليم و هي : من العام إلى الخاص

و من البسيط إلى المعقد و من

$$
\text { الوضيع الى الصعب. }
$$

كتاب "امثلتي ه" هناك ستة

$$
\text { أبواب. (أنظر إلى التالية) }
$$

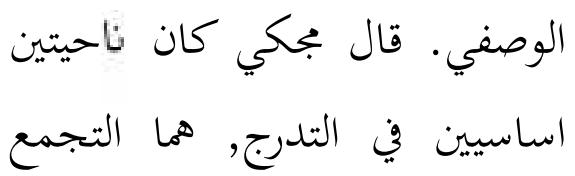

و و الرتيب (grouping)

قال جُكي يوجب التجمع أن

يسند بمبداء التجانسات

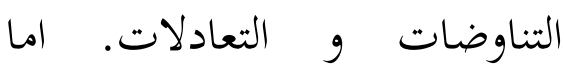

الرتيب، يوجب على الرتيب أن

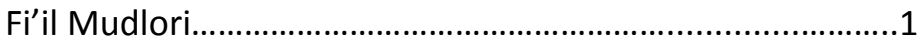

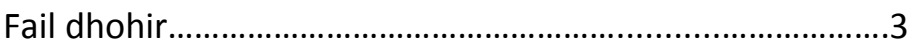

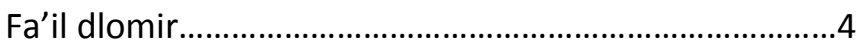

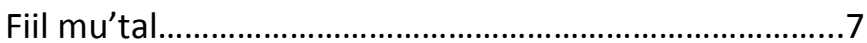

Wazan fiil mudlori.

يفعل،يفعل

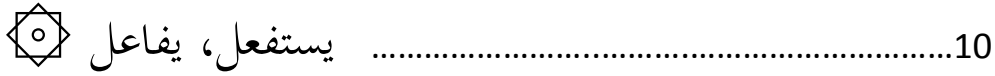

يفتعل، ينفعل

\&

Fa'il dlomir dari mudlori yang Diwali huruf ya'.....

Nun taukid.

.19

Mudlori yang dibaca nashab

Af'alul khomsah.

Mudlori' yang dibaca jazm 
Syarat dan jawab................................................................26

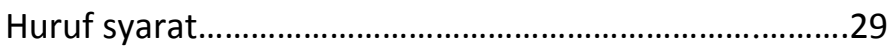

Fi'il amar...

.29

Fi'il amar yang mu'tal dari madly 3 huruf. .36

Qoidah-qoidah penting . .40

Khobar jumlah. 41

Bentuk-bentuk khobar .42

Shilah maushul .43

Hal dan shifat. .45

الخامس يبداء بفعل الأمر وبعده

فعل الأمر المعتال من فعل ماض

ثلاث الحروف. الباب السادس

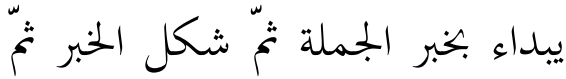

صلة المةوصول ثمّ حال و صفة.

رأت الباحثة بأنّ هذا الكتاب

يوافق بإطار النظري. الكتاب هو يستخدم تدرجين و هما التجمع و الرتيب.

عند التجمع، قال بكي

: يوجب التجمع أن يسند بمبداء

التجانسات و و

التعادلات. شكل التجانسات
الباب الأوّل، ينظم مؤلف

الكتاب المواد من مادة فعل المضارع و يستمر بفاعل ظاهر و يستمر بفاعل ضمير و يستمر بفعل معتال. ثم الباب الثاني، يبداء بأوزان فعل المضارع ثمّ فاعل ضمير من مضارع الذي يبدأ يِالياء، نون التوكيد. الباب الثالث يبداء بمضارع الذي يقرأ بالنصب تمّ م افعال الخمسة. الباب الرابع يبداء بمضارع الذي يقرأ بالجزم و يستمر بالشرط و الجواب ثم حرف شرط. الباب 
و من الوضيع الى الصعب. المبدأ

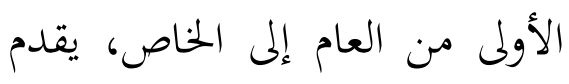
هذا الكتاب مثال في باب الأول العال العاب تعريف من فعل المضارع ثم يتبع

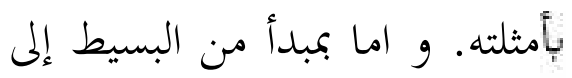

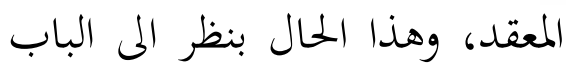

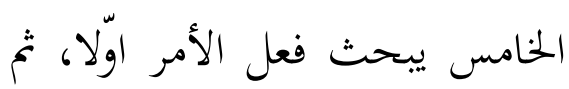
بعده يبحث المواد المعقد من قبله وهو فعل الأمر الذي معتالا. و اما لماد المغد فيله

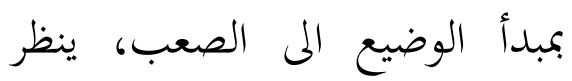

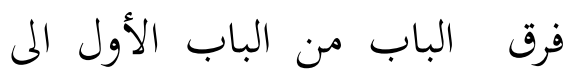

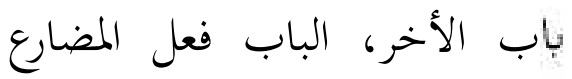
اولا، نعلم بأن فعل المضارع هو المابع شكل اساسي ليشكيل فعل غيره في علم الصرف.

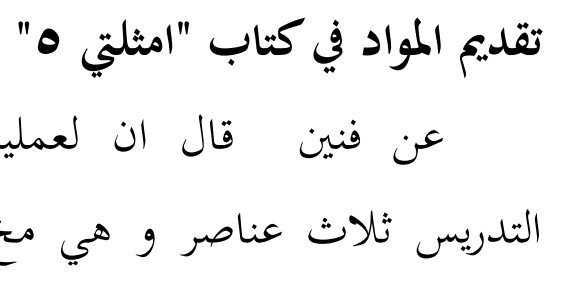
العنصر و تكامل العنصر و تقويم

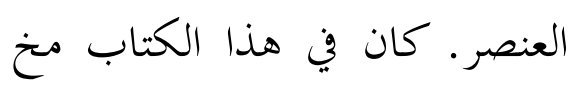
العنصر يتصور بالمواد المكتوبة فيه.
موجودة في هذا الكتاب كلهم يبحث في الفعل (فعل المضارع و فئاب

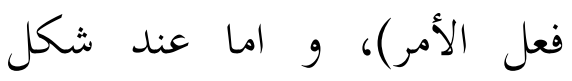
التناوضات في هذا الكتاب موجودة بفرق المبحب عن كل الباب( الباب الأول بالموضوع فعل المضارع، و المبحت البابل ابلاب

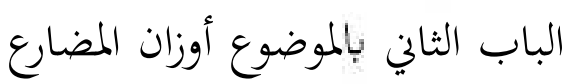

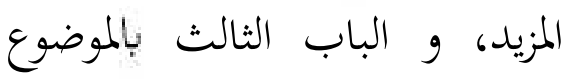
عوامل النواصب، و الباب الرابع بلموضوع عوامل جوازم، و الباب

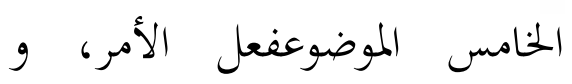
الباب السادس لموضوع مهمات).

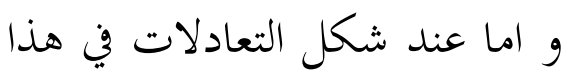
الكتاب موجودة بتطوير المواد في كل الباب مثال في باب الأول

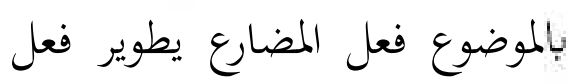

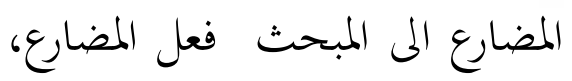

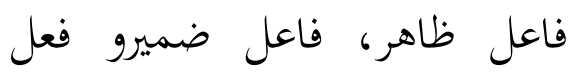
معتال عن فعل المضارع. عند الرتيب، بمبدأ : من العام إلى الخاص و من البسيط إلى المعقد 
باللغة العربية الى اللغة الإندونيسية و اللغة

$$
\begin{aligned}
& \text { الجاوية. (انظر الى التالية) } \\
& \text { المراجع }
\end{aligned}
$$

أرشد، أزهر. مدخل إلى طرق تعليم اللغة الأجنبية لمدرسي اللغة العربية.

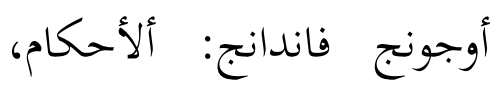

$$
.1991
$$

Analisis Data بوعين،

. Penelitian Kualitatif

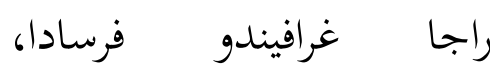

$$
\cdot r \cdot v
$$

Telaah Buku “ Hidayatul توفق، أحمد.

Wildan” Karya KH Muslich

Bin Abdurrahman (Analisis

. Perspektif Mackey) Materi

$$
\begin{aligned}
& \text { البحث العلمي6 } \\
& \text { الإسلامية الحكومية سونان }
\end{aligned}
$$

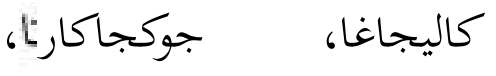

$$
\begin{aligned}
& \cdot r \cdot \Lambda \\
& \text { الحكيم، توفق. امثلتي ه. جيبارا: الفلاح }
\end{aligned}
$$

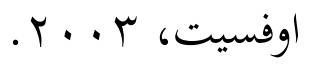

و تكامل العنصور في هذا الكتاب يتصور بصفحة الدافع ومرشد في تعليم لطلاب و لأساتذ. امّا تقويم العنصور في هذا الكتاب, هناك تدريبات و الممارسات المتنوعة. التقديم هو كيفية إيصال المواد الي طلاب اللغة ما يظهر علي صفحة من الكتاب. تقديم هذا المواد يعتمد علي أهداف التعليم و و مستوى كفاءة التلميذ. قال ججكي هناك اربع الإجراءات في (Mackey) التقديم: الإجراء الإجراء $\quad$ ostensive التصويري و الإجراء السياقي. طريقة التقديم في هذا الكتاب يستخدم ثلاث الإجراءات هم الإجراء التفضيلي و الإجراء السياقي والإجراء التصوير. الإجراء التفضيلي هو يشرح القاعدة بترجمة الشرح الى اللغة الأولى لطلابك، و في هذا الكتاب يشرح مؤلف الكتاب 


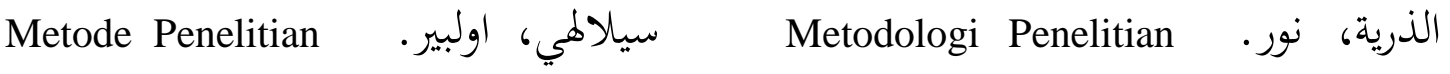

$$
\begin{aligned}
& \text { Sosial . Sosial Dan Pendidikan }
\end{aligned}
$$

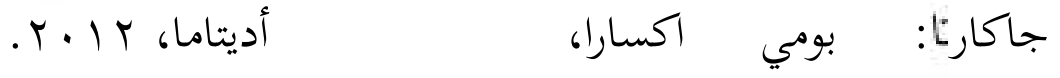

$$
\begin{aligned}
& \text { العارفين، زين. Evaluasi Pembelajaran. } \\
& \text { باندونج: بيماجا روسداكاريا، } \\
& . r \cdot 17 \\
& \text { عبد الحميد عبد الله، ناصر عبد الله } \\
& \text { الغالي. أساس إعداد الكتاب } \\
& \text { التعليمية لغير الناطقين بالعربية. } \\
& \text { القاهرة: دار الإعتصام، بغير }
\end{aligned}
$$

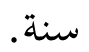

$$
\begin{aligned}
& \text { عبد الله، عبد الله الغالي و عبد الحميد. } \\
& \text { Menyusun Buku Ajar } \\
& \text { Bahasa Arab } \\
& \text { اكاديمييا فيرماتا، Y I · r. } \\
& \text { الغلاييني, مصطفى. جامع الدروس } \\
& \text { العربية. القاهرة: دار السلامم، } \\
& . r \cdot 1 r \\
& \text { متقين، امام. " دراسة تحليلية عن كتاب " } \\
& \text { أمثلة الجمل" تأليف الحاج امام } \\
& \text { زركشي و امام شيباني. } \\
& \text { رمة، جلال الدين. Metode Penelitian } \\
& \text { Komunikasi } \\
& \text { ريماجا روسداكاريا، r I ب.r. } \\
& \text { زائما، هيستي. " Analisis Penyajian } \\
& \text { Materi Mufrodat Pada } \\
& \text { Tingkat Madrasah } \\
& \text { 吕."Ibtidaiyyah } \\
& \text { الجامعة الإسلامية الحكومية }
\end{aligned}
$$

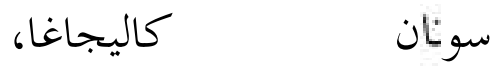

$$
\begin{aligned}
& \text { جوكجاكارنا، } 1 \text {.... } \\
& \text { Strategi Pembelajaran سانجايا، وينا. } \\
& \text { Berorientasi Standar Proses } \\
& \text { Pendidikan } \\
& \text { فرينادا ميدييا غروف، س ا • T. } \\
& \text { Penulisan Buku Teks سيتيفو. } \\
& \text { Pelajaran } \\
& \text { روسداكاريا، r I • r. }
\end{aligned}
$$




$$
\begin{aligned}
& \text { سيتائين فونوروغو فريس، } \\
& .4 .11 \\
& \text { و أخرون، رضية الدين. }
\end{aligned}
$$

Metodologi \& Strategi

Alternatif Pembelajaran

Bahasa Arab

فوستاكا رحلة غروف،

$$
. r \cdot 0
$$

و و أخرون، شتمس الدين. Metode

Penelitian Pendidikan

Bahasa

$$
\text { روسدا كاريا، } 0 \text { (ب. }
$$

و أخرون، عبد الحميد.Materi Media

Pembelajaran Bahasa Arab

, Metode Pendekatan

Strategi

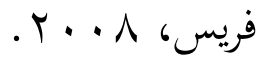

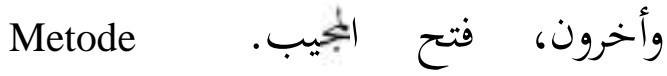

Permainan Edukatif Dalam

. Belajar Bahasa Arab

$$
\text { جوكجاكرتا: ديفا فريس6 }
$$

$$
\text { البحث العلمي. الجامعة المامية الحكومية بقونوروغو، }
$$

مُحَّم مخلص، عُمَّمَ منير. تحضير الكتاب

المدرسي للمستوى الإبتدئي.

فونوروغو: سيتاين فونوروغو

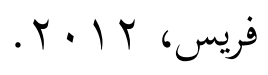

عُمَّم، مُعَّمَ عبد الخالق. اختبارات اللغة.

الراض: الجامعة الملك سعود،

$$
.8 \mid \leqslant 1 \text {. }
$$

مصلح، مسنور. Text Book Writing. جوكجاكرّا: الرّز ميدييا،

$$
.19
$$

Metode Penelitian نواوي، هدري.

Bidang Sosial

$$
\text { غاجه مادا فريس، V . . . . }
$$

Bahasa Arab نور سلام، يفريدا فطر.

Sejarah, Perkembangan,

Keistimewaan dan

Uونوروغو: Urgensinya 